\title{
El docente de bachillerato como sujeto-supuesto-a-saber de Lacan
}

\section{The high school teacher as Lacan's subject-supposed-to-know}

\author{
DOI: $10.46932 / \mathrm{sfjdv2n4-033}$
}

Received in: March 1st, 2021

Accepted in: May 30th, 2021

\author{
Adrián Flores Báez \\ Estudiante del Doctorado en Investigación e Intervención Educativa \\ Universidad Pedagógica Nacional U-211 \\ Prolongación de la 3 Sur y 121-A Poniente, Ex-Hacienda San Bartolo Coatepec, Puebla \\ E-mail: adflbz2@gmail.com \\ Mara Yazmín Morales Torres \\ Estudiante del Doctorado en Investigación e Intervención Educativa \\ Universidad Pedagógica Nacional U-211 \\ Prolongación de la 3 Sur y 121-A Poniente, Ex-Hacienda San Bartolo Coatepec, Puebla \\ E-mail: marayazminmoralestorres@gmail.com
}

\section{RESUMEN}

El presente trabajo es un intento de ligar la idea de sujeto-supuesto-a-saber de Lacan con lo educativo, donde el goce del docente de bachillerato es producto de la fuerza invisible del discurso de lo político sobre su actuar. El estudio se realizó en un bachillerato rural de la comunidad del Ocotal en Puebla, México, compuesto por tres docentes a quienes se entrevistaron de forma individual y posteriormente, después de ver 5 spots de la Reforma educativa se entrevistó de forma grupal. Los resultados mostraron que hay un choque entre los supuestos de los docentes (enfocados en la disciplina) y los de la Reforma (enfocados a la actualización permanente y dar lo mejor de sí).

Palabras clave: Bachillerato, Lacan, Reforma educativa, perfil docente, spot.

\section{ABSTRACT}

This paper is an attempt to link Lacan's idea of subject-supposed-to-know with education, where the enjoyment of the high school teacher is the product of the invisible force of the political discourse on his actions. The study was carried out in a rural high school in the community of Ocotal in Puebla, Mexico, made up of three teachers who were interviewed individually and later, after seeing 5 spots of the Educational Reform, they were interviewed as a group. The results showed that there is a clash between the assumptions of teachers (focused on the discipline) and those of the Reform (focused on permanent updating and giving their best).

Keywords: Highschool, Lacan, education reform, teacher profile, spot.

\section{INTRODUCCIÓN}

La identidad docente en la educación media superior mexicana ha sido un tema de constante debate, principalmente porque los que ejercen la docencia en este nivel educativo "llegaron a ella por 
diferentes motivos y circunstancias de vida. Hay quienes se formaron exprofeso para ello y hay quienes, de manera fortuita, azarosa o intencionada, optaron por ser docentes y descubrieron nuevas formas de ejercer una profesión, un oficio, un arte, un estilo de vida” (Mejía et al., 2021, p.1415). De esta manera, el magisterio manifiesta un sentimiento de frustración, acompañado de ansiedad por tratar de llevar a cabo las exceptivas de ser docente presentes en el perfil de la Reforma cuando la formación ajena a la docencia, choca con su deseo de ser vistos como "buenos maestros" por ellos mismos y el Otro: alumnado y los docentes con formación en la docencia. Además de lo anterior, otra circunstancia que afecta la identidad del magisterio es la carga horaria de las materias a impartir, ya que éstas, por cuestiones administrativas de ingreso, muchas de las veces no corresponden al perfil del docente, teniendo por resultado que existan materias que se desconocen y que irá aprendiendo a la par que el alumnado, esperando que un alumno no sepa más que éste, afectando así su discurso y sus acciones posteriores.

En consecuencia, la frustración y ansiedad que se manifiesta en el profesorado nos lleva a retomar el concepto de la "identidad emergente" de Hetrick (2010a) y aplicarlo en el docente de bachillerato. Ya que, para ser docente de bachillerato se tiene seguir las recomendaciones del perfil docente requeridas por la Reforma Integral de la Educación Media Superior (RIEMS). Y además, se tiene que considerar que en la práctica, aparte de estas recomendaciones intervienen los sistemas culturales del profesorado: lo que el docente cree es ser docente y las ideas de ser docente que se manejan en la mass media mexicana a través de spots publicitarios, realizados por el mismo gobierno mexicano, mostrando lo que es un docente según la RIEMS. De esta manera, los spots conciben identidades del docente, remarcando determinadas características y actuar de lo que se supone debe ser éste, teniendo consecuencias ontológicas que se derivan de esas identificaciones (Robertson, 1994) y de ahí que se considere su identidad como emergente.

\section{Metodología}

El estudio incluye entrevistas individuales con tres docentes ${ }^{1}$ ( 2 mujeres y 1 hombre) de un bachillerato en la comunidad rural del Ocotal en la Sierra Norte del Estado de Puebla, México y una entrevista grupal con los mismos tres participantes que se llevaron a cabo después de ver 5 spots de la Reforma educativa: (1) Spot Pacto por México; 2) Reforma educativa: reconocer a nuestros maestros, 3) Reforma Educativa: ¿Qué hijos le vamos a dejar a este país? 4) Reforma Educativa: Educación de Calidad y 5) Reforma Educativa: México con Formación. Estos spots fueron transmitidos continuamente por la televisión pública mexicana en el periodo de difusión de la RIEM (2013-2014), y ahora se encuentran fácilmente en los motores de búsqueda de internet y YouTube, lo que asegura que los spots anteriores

\footnotetext{
${ }^{1}$ Dos mujeres (Dm1 y Dm2) y un hombre (Dh). La formación de Dm1 es normalista y Dm2 y Dh es universitaria. Dm1 es directora y docente y Dm2 y Dh son docentes. Los años de experiencia como docentes es: Dm1: 10; Dm2: 5 y Dh: 5.
} 
considerando dotaron una representación de la cultura visual popular de los docentes y pueden funcionar como punto de partida para desplegar los deseos y fantasías pedagógicas inconscientes de los docentes.

Considerando lo anterior, el presente escrito se basa en la teoría psicoanalítica (Žižek, 1989 y Lacan, 2006), la cultura docente (Markgraf y Pavlik, 1998;) y se apoya con el análisis crítico del discurso ACD (van Dijk 1999) para el análisis de los referentes, con el que se construyeron dos categorías, las cuales se han nombrado como las fantasías pedagógicas más recurrentes que los docentes de bachillerato, ya que poseen y/o emplean un ideal de ser maestro. Tomando en cuenta que las fantasías pedagógicas son fantasías que involucran encuentros/intercambios pedagógicos entre dos o más personas (especialmente profesores y alumnos) dentro o fuera de un entorno educativo Hetrick (2010b), las fantasías pedagógicas a explorar son:

1) sujeto-supuesto-a-saber (Lacan, 1977)

2) la ilusión del docente

Estas fantasías pedagógicas tienen un peso en los docentes debido a la forma de asegurar gubernamentalmente la aplicación de la Reforma educativa, la cual fue a a través de cursos, diplomados y la condicionante del examen de permanencia en funciones docentes, donde la obtención de un puntaje bajo representa la capacitación urgente o la separación de la función docente. Considerando esto, se presentan a continuación estas fantasías.

Fantasía 1: El sujeto-supuesto-a-saber de Lacan

Dentro del contexto educativo el sujeto-supuesto-a-saber de Lacan debe entenderse como algo más que las palabras individuales o frases literales separadas por guiones. El concepto de este autor designa al que sabe, en este caso al docente, el que posee el conocimiento en el salón de clase y va ligado a la idea de transferencia, que desde la perspectiva lacaniana (¿Qué quiere el Otro de mí?, ¿qué me quiere?) se puede empatar con el amor que tiene el alumno por el maestro, cuando éste percibe en el maestro algo que no tiene (conocimiento). De esta forma el maestro es una figura de autoridad, es por tal el "sujeto-supuestoa-saber " y el estudiante presupone que este objeto está en el maestro "más que en sí mismo", creando la fantasía, el hechizo de transferencia, el objeto perdido (Jiménez, 2002).

Así, los docentes participantes en este estudio se consideran como sujeto-supuesto-a-saber, pues figuran como la autoridad que presupone sabe todo (en su caso el contenido de todas las materias) para el Otro: el alumnado. Además, los docentes han tenido una formación académica superior en comparación del alumnado. De esta manera, el conocimiento (académico) de los docentes es superior al del alumnado y por ello ocupan ese puesto. En este sentido, el supuesto del alumnado es que el docente sabe, y por ello está frente a ellos. Por tanto, el docente posee algo más que alumno no tiene, lo que pone a éste en un lugar de mayor conocimiento. Así, una vez que el docente se sitúa frente del alumnado (y no cualquier 
otro lado, como en las butacas o al fondo del salón) y es reconocido como el docente, el hechizo de transferencia comienza para algunos estudiantes. Para otros, tomará "algo de tiempo para que la transferencia se establezca" (Evans, 1996, p. 197), siendo completamente indiferente para el maestro o pensando cualquier cantidad de cosas potencialmente negativas sobre el maestro a primera vista. Pero, a medida que transcurre la clase, con su acción o con lo que dice el docente se denota algún conocimiento que capta la atención del alumnado incrédulo y comienza entonces, a encarnar lo que se supone debe de ser, estableciendo la transferencia.

La transferencia es, por lo tanto, importante para la educación y específicamente para el papel que juega el docente en el proceso de enseñanza-aprendizaje; y a menudo identificada como indistinguible del amor (Lacan, 1977), el concepto de transferencia ofrece una explicación razonable del respeto y el amor que los docentes tienen por ellos mismos como docentes porque "la transferencia puede entenderse como la propensión general a desplazar las relaciones pasadas a las experiencias actuales" (Robertson, 1994, p. 18).

Para aclarar lo anterior, se retoma la "la metáfora de la Madre" de Lacan (1957), en laque para designar a la mujer como introductora del lenguaje del niño, en el sentido de estructurar al sujeto a través del lenguaje y no en un sentido del papel de la mujer, se puede entender que la mujer (los padres) cumple el papel "del que sabe". Así, en el contexto de la educación, el alumnado hace la relación entre el profesorado y sus padres. Los compara bajo la idea "del que sabe" con referencia a sus relaciones pasadas, y entonces, el amor y el respeto hacia los padres se transfiere hacia el profesorado, el cual asume una posición autoritaria similar, pero ahora con un "tinte académico" en el alumnado, creándose así la fantasía de la materia supuesta a saber (el supuesto del docente) con respecto a la transferencia, lo que desflora: a) el tipo de maestro que los docente pueden desear / ser reconocidos como y b) los comportamientos y/o creencias resultantes de la trasferencia de cada docente.

Fantasía 2: La ilusión del docente

Schneider (2004) en su lectura sobre Lacan enfatiza el concepto de la ilusión, el cual considera que es constitutiva de la identidad y del lugar que cada cual ocupa en las relaciones sociales (Schneider, 2004, p.55). La ilusión, dice Lacan, es puesta en el sujeto en primera instancia por la Madre a través de los postulados: ella sabe, puede y quiere siempre lo que es bueno para éste. Por su parte, Schneider toma estos postulados y los transfiere a lo político, dándole al Estado la posición de la Madre y al ciudadano, la del niño. Así, el Estado sabe, el Estado puede y el Estado quiere. Desde esta perspectiva y empatándolo con lo educativo, se puede decir que el docente toma el lugar del niño por un lado ante el Estado, el cual da a conocer su ilusión de docente, a través de perfil y por otro, el docente ante el alumnado es el que sabe. Esta ilusión se puede ver en la campaña publicitaria a través de los discursos: a) en la mass media, 
(con un discurso sencillo y dirigido a la ciudadanía) y b) en el perfil de funciones docentes (con un discurso más especializado y dirigido al docente); las cuales exponemos a continuación.

a) La ilusión del docente desde los spots del Gobierno de la República

La importancia de los spots radica en el alcance de receptores que tuvieron, si es que consideramos que éstos fueron transmitidos por canales libres y de paga y repetidos, hasta dos veces, en un solo bloque publicitario, y en la Internet, convirtiendo a estos spots en un referente en todo aquel que lo ve (niños, jóvenes, adultos). Los supuestos sobre docente encontrados en los spots se muestran en la tabla siguiente:

Tabla 1. Supuestos del docente a través de los spots de la Reforma Educativa

\begin{tabular}{|c|c|c|}
\hline No. & spot & Supuestos \\
\hline \multirow[t]{3}{*}{1.} & Pacto por México & Si en la escuela hay maestros buenos, nuestros hijos tendrán el futuro asegurado \\
\hline & & Los maestros nos capacitamos para el futuro \\
\hline & & Mi maestra siempre está estudiando para enseñarnos mejor \\
\hline \multirow[t]{2}{*}{2.} & $\begin{array}{l}\text { Reforma educativa: } \\
\text { reconocer a nuestros } \\
\text { maestros }\end{array}$ & $\begin{array}{l}\text { Todos los días, sin importar la distancia, ni lo difícil del camino, ellos cumplen con su } \\
\text { misión de dar a millones de niños las herramientas para abrir las puertas a un futuro mejor. }\end{array}$ \\
\hline & & Educación de calidad para todos, de la mano de nuestros maestros \\
\hline 3. & $\begin{array}{l}\text { Reforma Educativa: } \\
\text { ¿Qué hijos le vamos a } \\
\text { dejar a este país? }\end{array}$ & $\begin{array}{l}\text { Si la Reforma Educativa, nos dará como beneficio el tener mejores maestros, mejores } \\
\text { instalaciones, condiciones de educación, maestros contentos, eh... maestros dedicados, } \\
\text { estoy con la reforma. }\end{array}$ \\
\hline 4. & $\begin{array}{l}\text { Reforma Educativa: } \\
\text { Educación de Calidad }\end{array}$ & $\begin{array}{l}\text { Todos los maestros tenemos un gran compromiso, con los jóvenes, con los niños. Porque } \\
\text { aparte de ser maestro, yo también soy padre y a mí también me gustaría que a mis hijos, } \\
\text { se les dé una educación de calidad, que el día de mañana, ellos puedan tener las armas } \\
\text { para salir a delante. }\end{array}$ \\
\hline \multirow[t]{3}{*}{5.} & $\begin{array}{l}\text { Reforma Educativa: } \\
\text { México con Formación }\end{array}$ & $\begin{array}{l}\text { Yo estoy a favor de la Reforma Educativa. Porque sé que nos van a evaluar para ser } \\
\text { mejores y vamos a estar los que tenemos que estar. }\end{array}$ \\
\hline & & Que se me reconozcan mis méritos, que se valore mi trabajo. \\
\hline & & $\begin{array}{l}\text { Así como nosotros vamos a ofrecer esa educación de calidad, necesitamos también calidad } \\
\text { para nosotros }\end{array}$ \\
\hline
\end{tabular}
Fuente: elaboración propia.

Como puede observarse el aspecto de capacitación, de estudio académico es el eje transversal que figura en los supuestos como un generador hacia un futuro mejor, es decir, un futuro que se asegura a través del estudio y preparación de los docentes y su reconocimiento.

b) La ilusión del docente desde el perfil de permanencia a funciones docentes

El perfil parte de lo establecido en el Artículo 12 de la Ley General del Servicio Profesional Docente (LGSPD) es una orientación de las “cualidades personales y profesionales" (SEP, 2018, p.9) que debe de tener quienes desempeñen la función docente. Los referentes se muestran bajo la idea de 5 dimensiones, las cuales se exponen con los supuestos del docente en la tabla 2. 
Tabla 2. Supuestos del docente a través del Perfil de permanencia a funciones docentes

\begin{tabular}{|c|c|c|}
\hline Dimensión & Descripción & Supuestos \\
\hline 1. & $\begin{array}{l}\text { Conocimientos para } \\
\text { el } \\
\text { Desempeño de la } \\
\text { función } \\
\text { Docente }\end{array}$ & $\begin{array}{l}\text { Adapta los conocimientos sobre la disciplina que imparte y los procesos de } \\
\text { enseñanza y aprendizaje de acuerdo con las características de los estudiantes y el } \\
\text { modelo basado en competencias. }\end{array}$ \\
\hline 2. & Práctica docente & $\begin{array}{l}\text { Planea los procesos de formación, enseñanza y aprendizaje y evaluación atendiendo } \\
\text { al modelo basado en competencias, y los ubica en contextos interno y externo }\end{array}$ \\
\hline 3. & $\begin{array}{l}\text { Desarrollo propio en } \\
\text { la función }\end{array}$ & Organiza y desarrolla su formación continua a lo largo de su trayectoria profesional \\
\hline 4. & $\begin{array}{l}\text { Vínculo con el } \\
\text { contexto escolar }\end{array}$ & $\begin{array}{l}\text { Vincula el contexto sociocultural y escolar con el proceso de enseñanza y } \\
\text { aprendizaje }\end{array}$ \\
\hline 5. & $\begin{array}{l}\text { Normativa y ética en } \\
\text { la función }\end{array}$ & $\begin{array}{l}\text { Construye ambientes de aprendizaje autónomo y colaborativo atendiendo el marco } \\
\text { normativo y ético }\end{array}$ \\
\hline
\end{tabular}
Fuente: elaboración propia.

Los supuestos, como puede observarse, apuntan hacia la preparación académica del docente en la materia que imparte y en procesos de enseñanza-aprendizaje principalmente, así como la búsqueda de su formación. En este sentido, haciendo la comparación con los supuestos de los spots de la Reforma encontramos que hay correspondencia en la forma en que tiene que ser el docente marcada por los supuestos comunes: capacitar y estar estudiando. La tabla 3 expone los significantes que dan los docentes del bachillerato a los supuestos de los spots y las dimensiones del perfil sobre el docente.

Tabla 3. Significantes comunes que los docentes dan a los spots de la Reforma Educativa y a las dimensiones del perfil de permanencia sobre el docente

\begin{tabular}{|l|l|rl|}
\hline Significante & Significado & Supuestos ( características que debe tener el docente) \\
\hline Docente & Compromiso & 1. & Dar lo mejor al alumnado para que aprenda \\
& Estudiar & 2. Mantenerse al día con las materias para saber que enseñar \\
& Prepararse & 3. Actualizarse en técnicas de enseñanza-aprendizaje \\
\hline
\end{tabular}

Fuente: elaboración propia.

Como se aprecia, las preocupaciones de los docentes se encuentran en que su preparación no ha sido la adecuada o la suficiente para cumplir los supuestos que marca el perfil, de ahí que la actualización, preparación y formación académica para mejorar su práctica docente sea el origen de su síntoma.

Reflexiones finales: El goce del docente de bachillerato

Para Jiménez (2002) la educación "ha pasado de la punición corporal al control provocado por el miedo donde más vale fingir que se enseñar"” (p.109). En este sentido, el docente de bachillerato tiene por miedo, la evaluación, y no por ser evaluado, sino por el esfuerzo que representa la misma en el cumplimiento del perfil deseado: cursos, talleres, modificar su práctica docente: llevar a cabo programas de desarrollo socioemocional, cumplir con las comisiones, desfiles, proyectos, evaluaciones, seguimiento, asesorías, vinculación, motivación. Lo anterior, hace que el docente motivado por el temor de perder el trabajo, busque la manera de llevar a cabo las actividades, lo cual despierta cierto interés por la mejora de 
su formación y de su práctica docente, haciendo que esa angustia por capacitarse se convierta en un goce, en una pulsión por saber, por tener una mejora y llegar a ser como el sujeto-supuesto-a-saber, a ser una ilusión del docente del perfil de la RIEMS:

- Docente $($ desde el Otro $)=$ disciplina = mantiene el grupo en silencio; cumple con lo que se tiene que entregar a dirección e impone su persona ante el alumnado.

- Docente $($ desde la mass media $)=$ premiado, con títulos y no politizado $=$ es reconocido por su trabajo académico; tiene una buena preparación académica y dedica su tiempo a la enseñanza.

- Docente $($ desde la Reforma Educativa $)=$ compromiso, estudiar y prepararse $=$ dar lo mejor al alumnado para que aprenda; mantenerse al día con las materias para saber que enseñar y actualizarse en técnicas de enseñanza-aprendizaje

Para concluir este texto, se puede decir que el goce del docente estriba en el choque de supuestos del docente. Por un lado, se tiene que desde el Otro se le da prioridad a la disciplina del alumnado y del profesorado, y por el otro, la mass media y la Reforma Educativa supone un docente que busca prepararse todo el tiempo y da lo mejor de sí. El cumplimiento de ésta ilusión, es por sí misma una proceso inacabado, ya que el docente al no dominar todas las materias, puesto que pasa por una formación inicial que lo ciñe, además de la que constantemente se actualizan los planes y programas curriculares por ciclo escolar y al tener a un alumnado que cada año cambia trayendo distintos elementos desde sus familias, el docente siempre tendrá necesidades y por tanto la capacitación se torna permanente, bajo la exigencia de que si no cumple entra la presión de perder su empleo. 


\section{REFERENCIAS}

Evans, D. (1996). An introductory dictionary of Lacanian psychoanalysis. London: Routledge

Guinsberg, E. (2003). "La influencia de los medios masivos en la formación del sujeto: una perspectiva psicoanalítica". En sicologia em Estudo, Maringá, 8(1), 3-12.

Hetrick, L. (2010a). "Exploring three pedagogical fantasies of becoming-teacher: A Lacanian and Deleuzo-Guattarian approach to unfolding the identity (re)formation of art student teachers". Dissertation Abstracts International, The Ohio State University

Hetrick, L. (2010b). "Exploring pedagogical fantasies in those learning to teach art". Visual Arts Research, 36(2), 38-48.

Jiménez, MA (2002). “Lacan y la educación”. En M. Gómez (Coord.) Teorías, epistemología y educación: debates contemporáneos. México: UNAM/Plaza y Valdes, pp. 89-122.

Lacan, J. (1955-1956) El seminario Libro 3 “Las Psicosis”, Paidós, Argentina, 2002.

(1956-1957). "El objeto fetiche. XI. El falo y la madre insaciable", en El Seminario, Libro 4, La relación de objeto, (1956-1957), Editorial Paidós, Buenos Aires, 1994, pp. 181-200.

(1977). “The Seminar. Book XI. The four fundamental concepts of psychoanalysis”. London: Hogarth Press and Institute of Psycho-Analysis.

(2006). "Ecrits: The first complete edition in English”. (Trans. B. Fink). New York: W.W. Norton \& Company.

Mejía, A., Fernández, T., Correa, A. y Sandoval J. (2021). "Propuesta para mejorar la calidad en la práctica docente de los niveles medio y superior del IPN (México), a través de la formación del personal académico". En South Florida Journal of Development, 2(2), 1414-1429.

Markgraf, S. y Pavlik, L. (1998). "Reel, metaphors for teaching”. Metaphor and Symbol, 13(4), 275-285.

Robertson, J. (1994). "Cinema and the politics of desire in teacher education". Dissertation Abstracts International, The University of Toronto.

Schneider, M. (2004). "El Estado como semblante”. En Y. Zarka (Dir.), Jacques Lacan. Psicoanálisis y política. Buenos Aires: Nueva Visión, pp. 51-64.

Van Dijk, T. (1999). Análisis Crítico del Discurso. Barcelona: Anthropos

Videos: Gobierno de la República [Gobierno de la República]. (04/04/2014-30/10/17): Spots [Archivos de video]. Recuperados de https://www.youtube.com/playlist?list=PLvtp80ozfi572UC3PWjvK2wWPwxo09eW (Consultados: 21/11/2018)

Žižek, S. (1989). The sublime object of ideology. United Kingdom: Verso. 